\title{
Truncated phase-space approach to polaron response
}

\author{
Dries Sels* and Fons Brosens ${ }^{\dagger}$ \\ Physics Department, University of Antwerp, Universiteitsplein 1, 2060 Antwerpen, Belgium
}

(Received 10 October 2013; published 17 January 2014)

\begin{abstract}
A method is presented to obtain the linear response coefficients of a system coupled to a bath. The method is based on a systematic truncation of the Liouville equation for the reduced distribution function. The firstorder truncation results are expected to be accurate in the low-temperature and weak-coupling regime. Explicit expressions for the conductivity of the Fröhlich polaron are obtained, and the discrepancy between the Kadanoff and the Feynman-Hellwarth-Iddings-Platzman mobility is elucidated.
\end{abstract}

DOI: 10.1103/PhysRevE.89.012124

PACS number(s): 05.30.-d, 71.38.-k

\section{INTRODUCTION}

Since its inception, the mobility of the Fröhlich polaron [1-3] has been the subject of many theoretical studies. For an excellent in-depth overview and discussion we refer to a textbook by Alexandrov and Devreese [4] and to lecture notes by Devreese [5]. A prominent approach was proposed by Feynman et al. [6] (hereafter referred to as FHIP), based on the path-integral formalism. This method is nonperturbative in the sense that no expansion in the coupling constant is assumed, but it is limited to first order in the applied electric field. However, in the asymptotic limit of weak electron-phonon coupling and low temperature, the FHIP polaron mobility differs by a factor of $3 /\left(2 \hbar \beta \omega_{L O}\right)$-with $\omega_{L O}$ the dispersionless longitudinal optical phonon frequency, and $\beta=1 /\left(k_{B} T\right)$ where $k_{B}$ is Boltzmann's constant and $T$ is the temperature-from the mobility which Kadanoff [7] found later on from the Boltzmann equation within the relaxation time approximation. As already pointed out by FHIP, the same factor of $3 /\left(2 \hbar \beta \omega_{L O}\right)$ appears in comparison with earlier results [8-10]. It has been argued in [11,12] that this discrepancy might be due to interchanging two limits (with both the frequency of the applied electric field and the electron-phonon coupling strength tending to zero). But this mathematical argument implicitly assumes the Kadanoff result to be valid, which we dispute.

In the present paper, we propose an alternative approach, based on the dynamics of the Wigner distribution function [13]. The methodology is basically inspired by the Feynman-Vernon influence functionals [14], rather than on Feynman's variational path-integral treatment of the ground-state energy of the polaron [15]. However, instead of considering the path integral for the wave function of a system, we contributed in [16] to a path-integral description of the Wigner distribution function. Concentrating on a particle that linearly interacts with a set of independent harmonic oscillators, the influence functional for the Wigner distribution function could be reduced to a double path integral in the path variables of the particle, if the oscillators are initially in thermodynamical equilibrium. In a subsequent paper [17] we derived a perturbation series for the propagator of the reduced Wigner function (i.e., the Wigner

\footnotetext{
*Corresponding author: dries.sels@uantwerpen.be

${ }^{\dagger}$ fons.brosens@uantwerpen.be
}

function for the particle of interest). By exactly resumming this series, we found a Dyson integral equation for the reduced propagator, from which the equation of motion for the reduced Wigner function could be derived. For general temperature and interaction strength, the resulting equation with a dressed propagator is still under investigation. We here concentrate on linear response at weak coupling and low temperature, in order to elucidate the discrepancy between the FHIP and the Kadanoff mobility.

The paper is organized as follows. In Sec. II we extract the assumptions and results from the papers $[16,17]$ which are relevant for our present purpose. In Sec. III we present an approximate, but systematically improvable, truncation method to derive the linear response coefficients from the equation of motion for the reduced Wigner function. We present a detailed discussion on the conductivity of the Frölich polaron in Sec. IV, after which we conclude in Sec. V. Supplementary information on the truncation scheme used is provided in Appendix A. Additional calculations on the relaxation time approximation and on the FHIP mobility are found in Appendixes B and C, respectively.

\section{REDUCED WIGNER FUNCTION FOR A GENERIC POLARON SYSTEM}

Consider the following generic polaron Hamiltonian:

$$
\begin{aligned}
H= & \frac{\mathbf{p}^{2}}{2 m}-e \mathbf{E}(t) \cdot \mathbf{x}+\sum_{k} \hbar \omega_{k}\left(b_{\mathbf{k}}^{\dagger} b_{\mathbf{k}}+\frac{1}{2}\right) \\
& +\sum_{\mathbf{k}}\left[\gamma(k) \exp (-i \mathbf{k} \cdot \mathbf{x}) b_{\mathbf{k}}^{\dagger}+\gamma^{*}(k) \exp (i \mathbf{k} \cdot \mathbf{x}) b_{\mathbf{k}}\right],
\end{aligned}
$$

where $(\mathbf{x}, \mathbf{p})$ represent the electron coordinate and momentum operator. It is coupled to some bosonic field $b_{\mathbf{k}}$ in an isotropic translationally invariant way, i.e., $\gamma(k)=\gamma(\mathbf{k})=\gamma(|\mathbf{k}|)$. Also the phonon frequency $\omega_{k}=\omega_{|\mathbf{k}|}$ is isotropic. The electron is subject to a time-dependent but homogeneous electric field $\mathbf{E}(t)$.

Because the system is translationally invariant in the absence of the field $\mathbf{E}(t)$, we suppose that the electron distribution is homogeneous, and that the phonon bath 
is initially in thermal equilibrium:

$$
f\left(\mathbf{r}, \mathbf{p},\left\{x_{\mathbf{k}}, p_{\mathbf{k}}\right\}, t=-\infty\right)=f(\mathbf{p}, t=-\infty) \prod_{\mathbf{k}} \frac{\tanh \frac{\beta \hbar \omega_{k}}{2}}{\pi \hbar} \exp \left[-\frac{\tanh \frac{\beta \hbar \omega_{k}}{2}}{\hbar \omega_{k}}\left(\frac{p_{\mathbf{k}}^{2}}{m}+m \omega_{k}^{2} x_{\mathbf{k}}^{2}\right)\right] .
$$

Knowledge of the (reduced) Wigner distribution function $f(\mathbf{p}, t)$ would allow calculation of the current density, and hence the conductivity $\sigma$,

$$
\begin{aligned}
\mathbf{J}(t) & =\frac{e}{m} \int \mathbf{p} f(\mathbf{p}, t) d \mathbf{p} \\
& =\int_{-\infty}^{t} \sigma\left(t-t^{\prime}\right) \mathbf{E}\left(t^{\prime}\right) d t^{\prime} .
\end{aligned}
$$

In general, $\sigma$ is a tensor but, due to the cylindrical symmetry of (2.1), it becomes diagonal. The Wigner-Liouville equation for the case of a phonon bath which initially is in thermal equilibrium, and for a general potential $V(\mathbf{x}, t)$, was derived in a recent paper [17]. For the electronic Hamiltonian $\frac{\mathbf{p}^{2}}{2 m}-e \mathbf{E}(t) \cdot \mathbf{x}$ under consideration here, the relevant equations (1.2)-(1.4) of Ref. [17] simplify to

$$
\begin{aligned}
& \left(\frac{\partial}{\partial t}+e \mathbf{E}(t) \cdot \frac{d}{d \mathbf{p}}\right) f(\mathbf{p}, t) \\
& =\sum_{\mathbf{k}} \frac{2|\gamma(k)|^{2}}{\hbar^{2}} \iiint \Theta\left(t^{\prime} \leqslant t\right)\left(\begin{array}{c}
\left(\begin{array}{c}
{\left[n_{B}\left(\omega_{k}\right)+1\right] \cos \left[\mathbf{k} \cdot\left(\mathbf{x}-\mathbf{x}^{\prime}\right)+\omega_{k}\left(t-t^{\prime}\right)\right]} \\
+n_{B}\left(\omega_{k}\right) \cos \left[\mathbf{k} \cdot\left(\mathbf{x}-\mathbf{x}^{\prime}\right)-\omega_{k}\left(t-t^{\prime}\right)\right]
\end{array}\right) \\
\times\left[K_{0}\left(\mathbf{x}, \mathbf{p}-\frac{\hbar \mathbf{k}}{2}, t \mid \mathbf{x}^{\prime}, \mathbf{p}^{\prime}+\frac{\hbar \mathbf{k}}{2}, t^{\prime}\right)-K_{0}\left(\mathbf{x}, \mathbf{p}+\frac{\hbar \mathbf{k}}{2}, t \mid \mathbf{x}^{\prime}, \mathbf{p}^{\prime}+\frac{\hbar \mathbf{k}}{2}, t^{\prime}\right)\right]
\end{array}\right) f\left(\mathbf{p}^{\prime}, t^{\prime}\right) d t^{\prime} d \mathbf{x}^{\prime} d \mathbf{p}^{\prime},
\end{aligned}
$$

with

$$
\begin{gathered}
K_{0}\left(\mathbf{x}, \mathbf{p}, t \mid \mathbf{x}^{\prime}, \mathbf{p}^{\prime}, t^{\prime}\right)=\delta\left(\mathbf{p}-\mathbf{p}^{\prime}-\int_{t^{\prime}}^{t} e \mathbf{E}(\sigma) \mathrm{d} \sigma\right) \delta\left(\mathbf{x}-\mathbf{x}^{\prime}-\frac{\mathbf{p}^{\prime}}{m}\left(t-t^{\prime}\right)-\int_{t^{\prime}}^{t} \frac{e \mathbf{E}(\sigma)}{m}(t-\sigma) d \sigma\right), \\
n_{B}\left(\omega_{k}\right)=1 /\left(e^{\beta \hbar \omega_{k}}-1\right) .
\end{gathered}
$$

Note that we have dropped the position dependence of the distribution function because both the initial state (2.2) and the electric field are homogeneous. In the absence of the electric field, the time evolution of the Wigner distribution becomes

$$
\frac{\partial f_{\mathbf{E}=\mathbf{0}}(\mathbf{p}, t)}{\partial t}=\sum_{\mathbf{k}} \frac{2|\gamma(k)|^{2}}{\hbar^{2}} \int_{0}^{\infty}\left(\begin{array}{c}
\left(\begin{array}{c}
{\left[n_{B}\left(\omega_{k}\right)+1\right] \cos \left[\left(\frac{(\mathbf{p}+\hbar \mathbf{k})^{2}-\mathbf{p}^{2}}{2 m \hbar}-\omega_{k}\right) s\right]} \\
+n_{B}\left(\omega_{k}\right) \cos \left[\left(\frac{(\mathbf{p}+\hbar \mathbf{k})^{2}-\mathbf{p}^{2}}{2 m \hbar}+\omega_{k}\right) s\right]
\end{array}\right) f_{\mathbf{E}=\mathbf{0}}(\mathbf{p}+\hbar \mathbf{k}, t-s) \\
-\left(\begin{array}{c}
{\left[n_{B}\left(\omega_{k}\right)+1\right] \cos \left[\left(\frac{(\mathbf{p}+\hbar \mathbf{k})^{2}-\mathbf{p}^{2}}{2 m \hbar}+\omega_{k}\right) s\right]} \\
+n_{B}\left(\omega_{k}\right) \cos \left[\left(\frac{(\mathbf{p}+\hbar \mathbf{k})^{2}-\mathbf{p}^{2}}{2 m \hbar}-\omega_{k}\right) s\right]
\end{array}\right) f_{\mathbf{E}=\mathbf{0}}(\mathbf{p}, t-s)
\end{array}\right) d s
$$

It seems unlikely that this integro-differential equation can be solved in closed form. Even a stationary solution $f_{\mathbf{E}=\mathbf{0}}^{\text {stat }}(\mathbf{p})$ in the absence of an electric field obeys a nontrivial integral equation. Using $\int_{0}^{\infty} \cos (a s) d s=\pi \delta(a)$, some elementary algebra reveals that, within the continuum limit, it satisfies the balance equation

$$
\int \Pi(\mathbf{p}+\hbar \mathbf{k} \rightarrow \mathbf{p}) f_{\mathbf{E}=\mathbf{0}}^{\text {stat }}(\hbar \mathbf{k}+\mathbf{p}) d \mathbf{k}=f_{\mathbf{E}=\mathbf{0}}^{\text {stat }}(\mathbf{p}) \Pi(\mathbf{p}),
$$

where we adopt a notation analagous to that introduced by Devreese and Evrard [18], and define

$$
\begin{gathered}
\Pi(\mathbf{p}+\hbar \mathbf{k} \rightarrow \mathbf{p})=\frac{V|\gamma(k)|^{2}}{(2 \pi)^{2} \hbar}\left(\begin{array}{c}
{\left[n_{B}\left(\omega_{k}\right)+1\right] \delta\left(\frac{(\mathbf{p}+\hbar \mathbf{k})^{2}-\mathbf{p}^{2}}{2 m}-\hbar \omega_{k}\right)} \\
+n_{B}\left(\omega_{k}\right) \delta\left(\frac{(\mathbf{p}+\hbar \mathbf{k})^{2}-\mathbf{p}^{2}}{2 m}+\hbar \omega_{k}\right)
\end{array}\right), \\
\Pi(\mathbf{p})=\int \Pi(\mathbf{p} \rightarrow \mathbf{p}+\hbar \mathbf{k}) d \mathbf{k} .
\end{gathered}
$$

Even this equation is hard to solve in its generality. One can, however, check by straightforward algebra that $f_{\mathbf{E}=\mathbf{0}}^{\text {stat }}(\mathbf{p}) \propto$ $\exp \left(-\beta \mathbf{p}^{2} / 2 m\right)$ satisfies Eq. (2.9). In order to elucidate the discrepancy between the mobility results of FHIP and Kadanoff, we limit the further discussion to linear response at weak coupling and low temperature. 


\section{LINEAR RESPONSE AT WEAK COUPLING AND LOW TEMPERATURE}

Limiting the discussion to first order in the electric field and to first order in $|\gamma(k)|^{2}$, the dependence on $\mathbf{E}$ of the reduced Wigner propagator (2.6) can be neglected, and the Wigner-Liouville equation (2.5) simplifies to

$$
\left(\frac{\partial}{\partial t}+e \mathbf{E}(t) \cdot \frac{d}{d \mathbf{p}}\right) f(\mathbf{p}, t)=\sum_{\mathbf{k}} \frac{2|\gamma(k)|^{2}}{\hbar^{2}} \int_{-\infty}^{t}\left(\begin{array}{c}
f(\mathbf{p}+\hbar \mathbf{k}, s)\left(\begin{array}{c}
{\left[n_{B}\left(\omega_{k}\right)+1\right] \cos \left[(t-s)\left(\mathbf{k} \cdot \frac{\mathbf{p}+\frac{\hbar \mathbf{k}}{2}}{m}-\omega_{k}\right)\right]} \\
+n_{B}\left(\omega_{k}\right) \cos \left[(t-s)\left(\mathbf{k} \cdot \frac{\mathbf{p}+\frac{\hbar \mathbf{k}}{2}}{m}+\omega_{k}\right)\right]
\end{array}\right) \\
-f(\mathbf{p}, s)\left(\begin{array}{c}
{\left[n_{B}\left(\omega_{k}\right)+1\right] \cos \left[(t-s)\left(\mathbf{k} \cdot \frac{\mathbf{p}+\frac{\hbar \mathbf{k}}{2}}{m}+\omega_{k}\right)\right]} \\
+n_{B}\left(\omega_{k}\right) \cos \left[(t-s)\left(\mathbf{k} \cdot \frac{\mathbf{p}+\frac{\hbar \mathbf{k}}{2}}{m}-\omega_{k}\right)\right]
\end{array}\right)
\end{array}\right) d s .
$$

It seems impossible to solve this highly non-Markovian initialvalue problem exactly.

Here we propose an approach which is inspired by the truncated Wigner approximation as, e.g., extensively discussed by Polkovnikov [19]. Its application to general coupling strength and arbitrary temperature is under current investigation. However, for sufficiently small electron-phonon coupling strength $\gamma(k)$ and sufficiently low temperature, the truncation after the first moment is justified, as argued in detail in Appendix A. It results in the following equation of motion (A4) for the current density:

$$
\frac{d \mathbf{J}(t)}{d t}+\int_{-\infty}^{t} \mathbf{J}(s) \chi(t-s) d s=\frac{e^{2}}{m} \mathbf{E}(t)
$$

where the memory function $\chi$ of the system is given by

$$
\begin{aligned}
\chi(t)= & t \sum_{\mathbf{k}} \frac{2|\gamma(k)|^{2}}{3 \hbar} \frac{\mathbf{k}^{2}}{m} \\
& \times\left(\begin{array}{c}
\left(n_{B}\left(\omega_{k}\right)+1\right) \sin \left[t\left(\frac{\hbar \mathbf{k}^{2}}{2 m}+\omega_{k}\right)\right] \\
+n_{B}\left(\omega_{k}\right) \sin \left[t\left(\frac{\hbar \mathbf{k}^{2}}{2 m}-\omega_{k}\right)\right]
\end{array}\right) .
\end{aligned}
$$

The definition (2.4) of the conductivity thus yields the following relation between the Laplace transform $\mathcal{L}(\sigma, \Omega)$ of the conductivity and the Laplace transform $\mathcal{L}(\chi, \Omega)$ of the memory function:

$$
\mathcal{L}(\sigma, \Omega)=\frac{e^{2}}{m} \frac{1}{\Omega+\mathcal{L}(\chi, \Omega)},
$$

from which one can, for example, immediately extract the (long-wavelength) optical absorption coefficient [20]

$$
\Gamma(\omega)=\frac{Z_{0}}{n} \operatorname{Re}[\mathcal{L}(\sigma, i \omega)]
$$

where $n$ is the crystal refractive index and $Z_{0}=\left(\epsilon_{0} c\right)^{-1}$ is the impedance of free space. Further results of course depend on the specifics of the system at hand. Here we apply the proposed model to the Fröhlich polaron.

\section{FRÖHLICH POLARON}

For the optical Fröhlich polaron one considers $\omega_{k}=\omega_{L O}$ to be constant. The coupling

$$
|\gamma(k)|^{2}=\frac{\hbar^{2} \omega_{L O}^{2}}{\mathbf{k}^{2}} \frac{4 \pi \alpha}{V} \sqrt{\frac{\hbar}{2 m \omega_{L O}}}
$$

scales with the dimensionless coupling constant $\alpha$. Then, in the continuum limit, the remaining integral in Eq. (3.3) is Gaussian and results in

$$
\begin{aligned}
\chi(t) & =\frac{2 \alpha \omega_{L O}^{2}}{3 \sqrt{2 \pi}}\left[\left[2 n_{B}\left(\omega_{L O}\right)+1\right] \frac{\cos \left(\omega_{L O} t\right)}{\sqrt{\omega_{L O} t}}-\frac{\sin \left(\omega_{L O} t\right)}{\sqrt{\omega_{L O} t}}\right] \\
& =\frac{\alpha \omega_{L O}^{2}}{3}\left\{\left[2 n_{B}\left(\omega_{L O}\right)+1\right] J_{-1 / 2}\left(\omega_{L O} t\right)-J_{1 / 2}\left(\omega_{L O} t\right)\right\},
\end{aligned}
$$

where $J_{ \pm 1 / 2}$ denotes the Bessel function of the first kind of order $\pm 1 / 2$. The Laplace transform [21] of $\chi$ is given by

$$
\begin{aligned}
\mathcal{L}(\chi, \Omega)= & \frac{\alpha \omega_{L O}}{3 \sqrt{\left(\frac{\Omega}{\omega_{L O}}\right)^{2}+1}} \\
& \times\left(\left(2 n_{B}\left(\omega_{L O}\right)+1\right) \sqrt{\sqrt{\left(\frac{\Omega}{\omega_{L O}}\right)^{2}+1}+\frac{\Omega}{\omega_{L O}}}\right. \\
& -\sqrt{\left.\sqrt{\left(\frac{\Omega}{\omega_{L O}}\right)^{2}+1}-\frac{\Omega}{\omega_{L O}}\right) .}
\end{aligned}
$$

Consequently the low-temperature dc conductivity (3.4) is

$\sigma_{d c}=\lim _{\Omega \rightarrow 0} \mathcal{L}(\sigma, \Omega)=\frac{3 e^{2}}{2 \alpha m \omega_{L O} n_{B}\left(\omega_{L O}\right)} \approx \frac{3 e^{2}}{2 \alpha m \omega_{L O}} e^{\beta \hbar \omega_{L O}}$.

It should immediately be noted that this result differs by a factor of 3 from that of Kadanoff [7] and by a factor of $\left(2 \hbar \beta \omega_{L O}\right)$ from that of FHIP [6], i.e.,

$$
\sigma_{d c}=\underset{\text { Kadanoff }}{3 \sigma_{d c}}=2 \hbar \beta \omega_{L O} \sigma_{\text {FHIP }} .
$$

The result is, however, in agreement with a prediction made by Los' [22], based on a Green's superoperator calculation of 
Kubo's formula. It was already argued by FHIP that in the $\Omega \rightarrow 0$ limit the full Boltzmann equation should be solved in order to get an accurate result for the dc mobility, an approximate solution of which was later provided by Kadanoff [7]. It was furthermore argued, in Refs. [11,12], that the $3 /\left(2 \hbar \beta \omega_{L O}\right)$ discrepancy was caused by an interchange of the $\Omega \rightarrow 0$ and $\alpha \rightarrow 0$ limits. One might wonder whether interchanging these limits gives different results in the current approach. The equation of motion for the current density was obtained by expanding the scattering term around $p \rightarrow 0$. One might guess that interchanging the limits by first taking the limit of $\Omega \rightarrow 0$, hence $t \rightarrow \infty$, and then the limit of $p \rightarrow 0$ will result in a similar difference. As argued in detail in Appendix B, this is not the case.

The discussion in Appendix B furthermore immediately explains the factor of 3 discrepancy between the present model and the result of Kadanoff. The in-scattering term in the Boltzmann equation, expressed in terms of the angular correlation factor in [7], is completely neglected by Kadanoff and dismissed as vanishingly small. But neglecting this inscattering violates particle number conservation. Within the present approach the in-scattering component is nonvanishing. The component linear in $E$ exactly subtracts $2 / 3\left(2 \alpha \omega_{L O}\right)$ from the inverse scattering rate, resulting in a mobility which is three times higher than the one calculated within the relaxation time approximation. It is clear that the present approach does not violate particle number conservation, and neither do the results of FHIP and Los'.

The additional $2 \hbar \beta \omega_{L O}$ difference with FHIP, however, remains to be explained. In Appendix $C$ we reexamine the FHIP approximation in the language of the distribution function rather than path integrals for the reduced density matrix. This illuminates the main problem in the FHIP approximation. First and foremost, unlike what is argued by FHIP, it is detrimental to assume an initial product state between the bath and the system for the evolution of the model. Although the true system will quickly thermalize to the temperature of the bath, the model system of FHIP does not thermalize, because it is completely harmonic and consequently fully integrable. In order to obtain a physical trial distribution one must assume that the complete model system was in thermal equilibrium instead of in a product state of the system with a thermal bath. Apart from this small change the analysis in Appendix $\mathrm{C}$ is completely in line with FHIP. The final low-temperature dc conductivity, however, reads

$$
\sigma_{d c}=\frac{3 e^{2}}{2 \alpha m^{*} \omega_{L O}} e^{\beta \hbar \omega_{L O}},
$$

where the effective mass $m^{*} / m=v^{2} / w^{2}$ is defined in terms of Feynman's variational parameters. Since $w \approx v$ and thus $m^{*} \approx m$ for sufficiently small $\alpha$, we recover the same result (4.4) as derived by our linearized equation of motion. It is clear that the present FHIP reanalysis does not have the spurious $2 \hbar \beta \omega_{L O}$ terms.

\section{CONCLUSION}

In conclusion, we have presented a method to obtain the conductivity of a generic polaron. In the low-temperature and weak-coupling regime a truncation after the first moment is justified and the conductivity is completely determined by a single memory function $\chi$. The method is used to study the conductivity of the Fröhlich polaron. It is found that the present approach results in a conductivity which is three times higher than the one predicted by Kadanoff and differs from that of FHIP by a factor $2 \hbar \beta \omega_{L O}$. Consequently we recover the result of Los' [22]. In order to elucidate the difference, we have reanalyzed the Boltzmann equation used by Kadanoff and the approach used by FHIP. Whereas the relaxation time approximation used by Kadanoff explicitly violates particle number conservation, the method developed by FHIP does not. The FHIP approximation, however, relies on an unphyiscal initial state for Feynman's polaron model. We find that a slightly modified version of both, which amends these two problems, accounts for their discrepancy.

\section{ACKNOWLEDGMENT}

The authors thank Professor J. T. Devreese for many stimulating discussions.

\section{APPENDIX A: TRUNCATED EQUATION OF MOTION}

Multiplying the Liouville equation (3.1) with $e \mathbf{p} / m$ and integrating out the momentum yields

$$
\begin{aligned}
& \int \frac{e \mathbf{p}}{m}\left(\frac{\partial}{\partial t}+e \mathbf{E}(t) \cdot \frac{d}{d \mathbf{p}}\right) f(\mathbf{p}, t) d \mathbf{p} \\
& =\sum_{\mathbf{k}} \frac{2|\gamma(k)|^{2}}{\hbar^{2}} \int_{-\infty}^{t}\left(\begin{array}{c}
\int \frac{e \mathbf{p}}{m} f(\mathbf{p}-\hbar \mathbf{k}, s)\left(\begin{array}{c}
{\left[n_{B}\left(\omega_{k}\right)+1\right] \cos \left[(t-s)\left(\mathbf{k} \cdot \frac{\mathbf{p}-\frac{\hbar \mathbf{k}}{2}}{m}+\omega_{k}\right)\right]} \\
+n_{B}\left(\omega_{k}\right) \cos \left[(t-s)\left(\mathbf{k} \cdot \frac{\mathbf{p}-\frac{\hbar \mathbf{k}}{2}}{m}-\omega_{k}\right)\right]
\end{array}\right) d \mathbf{p} \\
-\int \frac{e \mathbf{p}}{m} f(\mathbf{p}, s)\left(\begin{array}{c}
{\left[n_{B}\left(\omega_{k}\right)+1\right] \cos \left[(t-s)\left(\mathbf{k} \cdot \frac{\mathbf{p}+\frac{\hbar \mathbf{k}}{2}}{m}+\omega_{k}\right)\right]} \\
+n_{B}\left(\omega_{k}\right) \cos \left[(t-s)\left(\mathbf{k} \cdot \frac{\mathbf{p}+\frac{\hbar \mathbf{k}}{2}}{m}-\omega_{k}\right)\right]
\end{array}\right) d \mathbf{p}
\end{array}\right) d s
\end{aligned}
$$

Taking the expression (2.3) for the current density into account, the left-hand side can be calculated directly. After the substitution $\mathbf{p}-\hbar \mathbf{k} \rightarrow \mathbf{p}$ in the first term on the right-hand side, one is left with

$$
\frac{d \mathbf{J}(t)}{d t}-\frac{e^{2}}{m} \mathbf{E}(t)=\sum_{\mathbf{k}} \frac{2|\gamma(k)|^{2}}{\hbar} \mathbf{k} \frac{e}{m} \int_{-\infty}^{t} \int f(\mathbf{p}, s)\left(\begin{array}{c}
{\left[n_{B}\left(\omega_{k}\right)+1\right] \cos \left[(t-s)\left(\frac{\mathbf{k} \cdot \mathbf{p}}{m}+\frac{\hbar k^{2}}{2 m}+\omega_{k}\right)\right]} \\
+n_{B}\left(\omega_{k}\right) \cos \left[(t-s)\left(\frac{\mathbf{k} \cdot \mathbf{p}}{m}+\frac{\hbar k^{2}}{2 m}-\omega_{k}\right)\right]
\end{array}\right) d \mathbf{p} d s .
$$


Using the $\mathbf{k} \leftrightarrow-\mathbf{k}$ symmetry results in

$$
\begin{aligned}
\frac{d \mathbf{J}(t)}{d t}-\frac{e^{2}}{m} \mathbf{E}(t)= & -\sum_{\mathbf{k}} \frac{2|\gamma(k)|^{2}}{\hbar} \mathbf{k} \int_{-\infty}^{t}\left(\begin{array}{c}
n_{B}\left(\omega_{k}\right) \sin \left[\left(\frac{\hbar k^{2}}{2 m}-\omega_{k}\right)(t-s)\right] \\
+\left[n_{B}\left(\omega_{k}\right)+1\right] \sin \left[\left(\frac{\hbar k^{2}}{2 m}+\omega_{k}\right)(t-s)\right]
\end{array}\right) \\
& \times \frac{e}{m} \int f(\mathbf{p}, s) \sin \left(\frac{\mathbf{k} \cdot \mathbf{p}}{m}(t-s)\right) d \mathbf{p} d s .
\end{aligned}
$$

Since the current density (2.3) is of order $\mathbf{E}$, the dominant contribution in the last line of this equation is provided by the small momenta. It thus seems reasonable to expand the sine function:

$$
\begin{aligned}
\frac{e}{m} \int f(\mathbf{p}, s) \sin \left(\frac{\mathbf{k} \cdot \mathbf{p}}{m}(t-s)\right) d \mathbf{p} & =\frac{e}{m} \int f(\mathbf{p}, s)\left[\frac{\mathbf{k} \cdot \mathbf{p}}{m}(t-s)-\left(\frac{\mathbf{k} \cdot \mathbf{p}}{m}\right)^{3} \frac{(t-s)^{3}}{6}+\cdots\right] d \mathbf{p} \\
& =(t-s) \frac{\mathbf{k} \cdot \mathbf{J}(s)}{m}-\frac{(t-s)^{3}}{6} \frac{e}{m} \int f(\mathbf{p}, s)\left(\frac{\mathbf{k} \cdot \mathbf{p}}{m}\right)^{3} d \mathbf{p}+\cdots
\end{aligned}
$$

For general coupling strength $\gamma(k)$ and temperature, this expansion seems not very useful. Indeed, the Wigner function broadens with increasing temperature. Furthermore, for strong coupling the initial phonon states are better described by displaced and broadened Gaussian wave functions, as shown in the derivation of the optical absorption of polarons in $[23,24]$. The change in the initial phonon state will affect the influence phase [16] and consequently the self-energy [17]. A dressed propagator will replace the free-particle propagator (2.6). The extension of the present result to strong coupling will be a topic of forthcoming work.

However, in the present paper we were mainly concerned with the discrepancy between the FHIP result and the Kadanoff result for small electron-phonon coupling and low temperature. In that case, neither the electron-phonon coupling nor the temperature is able to broaden the distribution function substantially. Therefore, for $\gamma(k)$ and $T$ sufficiently small, one might truncate the expansion to the first moment, which results in

$$
\frac{d \mathbf{J}(t)}{d t}-\frac{e^{2}}{m} \mathbf{E}(t) \approx-\sum_{\mathbf{k}} \frac{2|\gamma(k)|^{2}}{\hbar} \mathbf{k} \int_{-\infty}^{t}\left(\begin{array}{c}
{\left[n_{B}\left(\omega_{k}\right)+1\right] \sin \left[(t-s)\left(\frac{\hbar k^{2}}{2 m}+\omega_{k}\right)\right]} \\
+n_{B}\left(\omega_{k}\right) \sin \left[(t-s)\left(\frac{\hbar k^{2}}{2 m}-\omega_{k}\right)\right]
\end{array}\right)(t-s) \frac{\mathbf{k} \cdot \mathbf{J}(s)}{m} d s .
$$

Note that one can systematically improve the result [19] by the equations of motion for the higher moments.

\section{APPENDIX B: RELAXATION TIME APPROXIMATION}

The purpose of this appendix is to explain the discrepancy in (4.5) by a factor of 3 between the dc conductivity of the Fröhlich polaron which we derived in (4.4), as compared to the Kadanoff result [7]. We thus consider the linearized Liouville equation (3.1) for the reduced Wigner function. Using $\int_{-\infty}^{t} \cos [(t-s) a] d s=\pi \delta(a)$ one easily derives that its stationary version is a Boltzmann equation:

$$
e \mathbf{E} \cdot \frac{d f(\mathbf{p})}{d \mathbf{p}}=-\Pi(\mathbf{p}) f(\mathbf{p})+\int \Pi(\mathbf{p}+\hbar \mathbf{k} \rightarrow \mathbf{p}) f(\mathbf{p}+\hbar \mathbf{k}) d \mathbf{k},
$$

with $\Pi(\mathbf{p}+\hbar \mathbf{k} \rightarrow \mathbf{p})$ and $\Pi(\mathbf{p})$ defined in (2.10) and (2.11).

Because the unperturbed reduced Wigner distribution function at sufficiently low temperature peaks around $\mathbf{p}=\mathbf{0}$, one might argue that the dominant term in the right-hand side is given by $-f(\mathbf{p}) \lim _{\mathbf{p} \rightarrow \mathbf{0}} \Pi(\mathbf{p})$, which gives rise to a relaxation time approximation (RTA):

$$
e \mathbf{E} \cdot \frac{d f(\mathbf{p})}{d \mathbf{p}} \approx-\frac{f(\mathbf{p})}{\tau} \quad \text { with } \quad \tau=\frac{1}{\lim _{\mathbf{p} \rightarrow \mathbf{0}} \Pi(\mathbf{p})} .
$$

The first moment of this equation with respect to $\mathbf{p}$, taking (2.3) into account, then immediately leads to

$$
\mathbf{J}=\lim _{\mathbf{p} \rightarrow 0} \frac{e^{2} / m}{\Pi(\mathbf{p})} \mathbf{E} ; \quad \text { hence } \quad \begin{aligned}
& \sigma_{d c} \\
& R T A
\end{aligned}=\lim _{\mathbf{p} \rightarrow 0} \frac{e^{2} / m}{\Pi(\mathbf{p})} .
$$

For the Fröhlich polaron, with the constant frequency $\omega_{k}=\omega_{L O}$ and the electron-phonon coupling (4.1), the corresponding function $\Pi_{\text {Fröhlich }}(\mathbf{p})$ can easily be calculated in closed form:

$$
\Pi_{\text {Fröhlich }}(\mathbf{p})=2 \alpha \omega_{L O} \frac{\sqrt{2 m \hbar \omega_{L O}}}{p}\left(\begin{array}{c}
{\left[n_{B}\left(\omega_{L O}\right)+1\right] \Theta\left(\hbar \omega_{L O}<\frac{p^{2}}{2 m}\right) \operatorname{arccosh} \frac{p}{\sqrt{2 m \hbar \omega_{L O}}}} \\
+n_{B}\left(\omega_{L O}\right) \operatorname{arcsinh} \frac{p}{\sqrt{2 m \hbar \omega_{L O}}}
\end{array}\right) .
$$

This simple relaxation time approximation thus immediately gives the Kadanoff conductivity for the Fröhlich polaron:

$$
\underset{\text { Kadanoff }}{\sigma_{d c}}=\lim _{\mathbf{p} \rightarrow \mathbf{0}} \frac{e^{2} / m}{\Pi_{\text {Fröhlich }}(\mathbf{p})} \approx \frac{1}{2} \frac{e^{2}}{m \alpha \omega_{L O}} e^{\beta \hbar \omega_{L O}} .
$$


However, the neglect of the integral term in (B1) is an unwarranted approximation, essentially because it violates the particle number conservation. Indeed, consider the first moment of (B1) with respect to p:

$$
e \mathbf{E}=\int \mathbf{p} \Pi(\mathbf{p}) f(\mathbf{p}) d \mathbf{p}-\iint \mathbf{p} \Pi(\mathbf{p}+\hbar \mathbf{k} \rightarrow \mathbf{p}) f(\mathbf{p}+\hbar \mathbf{k}) d \mathbf{k} d \mathbf{p} .
$$

By the substitution $\mathbf{p}+\hbar \mathbf{k} \rightarrow \mathbf{p}$ in the last term, interchanging $\mathbf{k} \leftrightarrow-\mathbf{k}$, and using the definition (3.3), the terms in $\Pi$ (p) cancel against each other, and one is left with

$$
e E=-\mathbf{1}_{E} \cdot \iint \hbar \mathbf{k} \Pi(\mathbf{p} \rightarrow \mathbf{p}+\hbar \mathbf{k}) f(\mathbf{p}) d \mathbf{k} d \mathbf{p},
$$

which shows that the in-scattering rate cannot be neglected.

At sufficiently low temperature, the distribution function peaks at $\overline{\mathbf{p}}=m \mathbf{J} / e$ which is indeed near $\mathbf{p}=\mathbf{0}$ since $\overline{\mathbf{p}} \propto \mathbf{E} \rightarrow \mathbf{0}$. Replacing $f(\mathbf{p})$ by $\delta(\mathbf{p}-m \mathbf{J} / e)$ then gives

$$
e E=-\mathbf{1}_{E} \cdot \int \hbar \mathbf{k} \Pi\left(\frac{m \mathbf{J}}{e} \rightarrow \frac{m \mathbf{J}}{e}+\hbar \mathbf{k}\right) d \mathbf{k} .
$$

For the Fröhlich polaron (4.1), the evaluation of this integral is elementary and results in

$$
\begin{aligned}
& e E=m \omega_{L O} \alpha \sqrt{2} \sqrt{\frac{\hbar \omega_{L O}}{m}} \frac{2 e^{2}}{m J^{2}}
\end{aligned}
$$

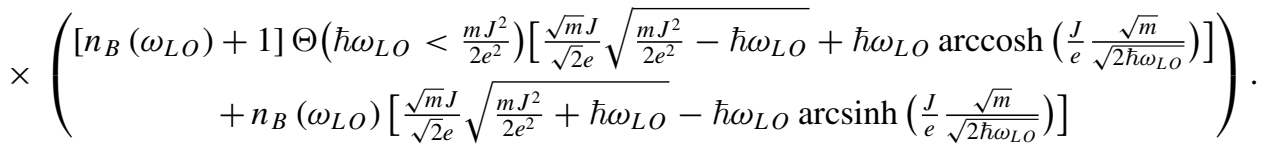

Keeping linear response in mind, it is obvious that this expression is needed only to first order in $J=O(E)$, such that the emission term does not contribute at sufficiently low temperature. The result is

$$
e E=\frac{2}{3} m \omega_{L O} \alpha n_{B}\left(\omega_{L O}\right) \frac{J}{e}+O\left(J^{3}\right)
$$

which is fully consistent with the conductivity (4.4) derived above.

\section{APPENDIX C: FHIP APPROXIMATION WITH DISTRIBUTION FUNCTION}

In this appendix we present a calculation in the spirit of the FHIP approximation but using our phase-space approach. It was shown in [17] how the path integral for the reduced Wigner function leads to the Liouville equation (2.5). The path integral for the reduced Wigner function is just the Weyl transform of the path integral for the density matrix used by FHIP. The basic approach of FHIP is to expand the action around Feynman's linear polaron model, rather than around the free particle. In terms of the distribution function this means that

$$
f(\mathbf{p}, t)=f_{0}(\mathbf{p}, t)+f_{1}(\mathbf{p}, t)
$$

where $f_{0}$ is a variational time-dependent Wigner function which can be found by propagating the initial distribution along a certain linear model, which we are so far free to choose. Similarly to the linear response at weak coupling (i.e., to first order in the deviation from the free particle), we now consider linear response to first order in the deviation from the Feynman polaron model, which means that

$$
\left(\frac{\partial}{\partial t}+e \mathbf{E} \cdot \nabla_{p}\right) f_{1}(\mathbf{p}, t)=g_{0}(\mathbf{p}, t),
$$

where $g_{0}(\mathbf{p}, t)$, apart from the time evolution of $f_{0}$, is the right-hand side of (3.1) with $f$ replaced by $f_{0}$ :

$$
\begin{aligned}
g_{0}(\mathbf{p}, t)= & -\left(\frac{\partial}{\partial t}+e \mathbf{E}(t) \cdot \nabla\right) f_{0}(\mathbf{p}, t) \\
& +\sum_{\mathbf{k}} \frac{2|\gamma(k)|^{2}}{\hbar^{2}} \int_{-\infty}^{t}\left(\begin{array}{c}
f_{0}(\mathbf{p}+\hbar \mathbf{k}, s)\left(\begin{array}{c}
{\left[n_{B}\left(\omega_{k}\right)+1\right] \cos \left[(t-s)\left(\mathbf{k} \cdot \frac{\mathbf{p}+\frac{\hbar \mathbf{k}}{2}}{m}-\omega_{k}\right)\right]} \\
+n_{B}\left(\omega_{k}\right) \cos \left[(t-s)\left(\mathbf{k} \cdot \frac{\mathbf{p}+\frac{\hbar \mathbf{k}}{2}}{m}+\omega_{k}\right)\right]
\end{array}\right) \\
-f_{0}(\mathbf{p}, s)\left(\begin{array}{c}
{\left[n_{B}\left(\omega_{k}\right)+1\right] \cos \left[(t-s)\left(\mathbf{k} \cdot \frac{\mathbf{p}+\frac{\hbar \mathbf{k}}{2}}{m}+\omega_{k}\right)\right]} \\
+n_{B}\left(\omega_{k}\right) \cos \left[(t-s)\left(\mathbf{k} \cdot \frac{\mathbf{p}+\frac{\hbar \mathbf{k}}{2}}{m}-\omega_{k}\right)\right]
\end{array}\right) d s .
\end{array}\right)
\end{aligned}
$$


The time dependence of the distribution function $f_{1}$ follows the classical equation of motion, and consequently

$$
f_{1}(\mathbf{p}, t)=\int_{-\infty}^{t} g_{0}\left(\mathbf{p}-\int_{t^{\prime}}^{t} e \mathbf{E}(s) d s, t^{\prime}\right) d t^{\prime} .
$$

Because of the particle number conservation of the trial distribution, and due to the linearity of the classical equation of motion, the expected current density of the perturbation around the model becomes

$$
\mathbf{J}_{1}(t)=\frac{e}{m} \int \mathbf{p} f_{1}(\mathbf{p}, t) \mathrm{d} \mathbf{p}=\frac{e}{m} \int_{-\infty}^{t} \int \mathbf{p} g_{0}\left(\mathbf{p}, t^{\prime}\right) d \mathbf{p} d t^{\prime}
$$

The total current density is consequently given by

$$
\mathbf{J}(t)=\mathbf{J}_{0}(t)+\mathbf{J}_{1}(t)
$$

where $\mathbf{J}_{0}(t)$ is the current density of the model distribution function. In terms of Feynman's variational parameters $w$ and $v$, Feynman's model distribution function reads

$$
\begin{aligned}
f_{0}(\mathbf{p}, t)= & \left(\frac{\beta}{2 m \pi}\right)^{3 / 2} \exp \left[-\frac{\beta}{2 m}\left(\mathbf{p}-\frac{w^{2}}{v^{2}} \int_{-\infty}^{t} e \mathbf{E}(s) d s\right.\right. \\
& \left.\left.-\frac{v^{2}-w^{2}}{v^{2}} \int_{-\infty}^{t} e \mathbf{E}(s) \cos v(t-s) d s\right)^{2}\right],
\end{aligned}
$$

provided we assume the model to be initially in canonical equilibrium at an effective temperature equal to the real temperature $\beta^{-1}$. At this point the present discussion differs from that of FHIP, where the initial state of the model is assumed to be a product state of the oscillators with the particle. It is argued by FHIP that the product state ansatz is admissible because "In the past only the oscillators were in thermal equilibrium at $\beta^{-1}$. As a result of the coupling the system will come very quickly to thermal equilibrium at the same temperature" [6]. Although this might be true for the real system, it does not apply to the model. Because of the linearity of the model it will never thermalize. Consequently, the reduced model distribution function will endlessly oscillate even in the absence of an electric field. In contrast, the present model distribution is the exact stationary distribution of the reduced Liouville equation in the absence of an electric field [17]. It should, however, also be noted that, as a consequence of the same linearity, the expected model current density

$$
\begin{aligned}
\mathbf{J}_{0}(t)= & \frac{w^{2}}{v^{2}} \int_{-\infty}^{t} \frac{e^{2} \mathbf{E}(s)}{m} d s+\frac{v^{2}-w^{2}}{v^{2}} \\
& \times \int_{-\infty}^{t} \frac{e^{2} \mathbf{E}(s)}{m} \cos v(t-s) d s
\end{aligned}
$$

is not affected by the change in initial state, in contrast to the correction $\mathbf{J}_{1}(t)$. From the definition (2.4) of the conductivity, we furthermore find the following expression for the Laplace transform $\mathcal{L}\left(\sigma_{0}, \Omega\right)$ of the model conductivity:

$$
\mathcal{L}\left(\sigma_{0}, \Omega\right)=\frac{e^{2}}{m}\left(\frac{w^{2}}{v^{2}} \frac{1}{\Omega}+\frac{v^{2}-w^{2}}{v^{2}} \frac{\Omega}{v^{2}+\Omega^{2}}\right) .
$$

The first-order correction $\mathbf{J}_{1}(t)$ consists of two parts, one that scales with the coupling constant and one that does not.
The latter is given by

$$
\begin{aligned}
\mathbf{J}_{1,0}(t) & =-\int_{-\infty}^{t} \int e \mathbf{p}\left(\frac{\partial}{\partial t}+e \mathbf{E}(t) \cdot \nabla_{p}\right) f_{0}\left(\mathbf{p}, t^{\prime}\right) d \mathbf{p} d t^{\prime} \\
& =\frac{v^{2}-w^{2}}{v^{2}}\left[\int_{-\infty}^{t} \frac{e^{2} \mathbf{E}(s)}{m} d s\right. \\
& \left.-\int_{-\infty}^{t} \frac{e^{2} \mathbf{E}(s)}{m} \cos v(t-s) d s\right] .
\end{aligned}
$$

The coupling-dependent part leads to

$$
\begin{aligned}
\mathbf{J}_{1,1}(t)= & \frac{e}{m} \int_{-\infty}^{t} d t^{\prime} \int_{-\infty}^{t^{\prime}} d s \sum_{\mathbf{k}} \frac{2|\gamma(k)|^{2}}{\hbar} \mathbf{k} \int f_{0}(\mathbf{p}, s) \\
& \times\left(\begin{array}{c}
{\left[n_{B}\left(\omega_{k}\right)+1\right] \cos \left[\left(t^{\prime}-s\right)\left(\frac{\mathbf{k} \cdot \mathbf{p}}{m}+\frac{\hbar k^{2}}{2 m}+\omega_{k}\right)\right]} \\
+n_{B}\left(\omega_{k}\right) \cos \left[\left(t^{\prime}-s\right)\left(\frac{\mathbf{k} \cdot \mathbf{p}}{m}+\frac{\hbar k^{2}}{2 m}-\omega_{k}\right)\right]
\end{array}\right) \\
& \times d \mathbf{p},
\end{aligned}
$$

which within linear response, hence up to $O(E)$, simplifies to

$$
\mathbf{J}_{1,1}(t)=-\int_{-\infty}^{t} d t^{\prime} \int_{-\infty}^{t^{\prime}} d s \chi_{\beta}\left(t^{\prime}-s\right) \mathbf{J}_{0}(s)
$$

with

$$
\begin{aligned}
\chi_{\beta}(t)= & t \sum_{\mathbf{k}} \frac{2|\gamma(k)|^{2}}{3 \hbar} \frac{\mathbf{k}^{2}}{m}\left(\begin{array}{c}
{\left[n_{B}\left(\omega_{k}\right)+1\right] \sin \left[t\left(\frac{\hbar \mathbf{k}^{2}}{2 m}+\omega_{k}\right)\right]} \\
+n_{B}\left(\omega_{k}\right) \sin \left[t\left(\frac{\hbar \mathbf{k}^{2}}{2 m}-\omega_{k}\right)\right]
\end{array}\right) \\
& \times \exp \left(-\frac{\mathbf{k}^{2}}{2 m \beta} t^{2}\right) .
\end{aligned}
$$

Note that $\lim _{\beta \rightarrow \infty} \chi_{\beta}(t)=\chi(t)$, where $\chi(t)$ is the memory function obtained by truncating the equation of motion for the current density, as explained in Appendix A. Consequently the low-temperature, linear response, current density up to first order around the Feynman polaron model is

$$
\mathbf{J}(t)=\int_{-\infty}^{t} \frac{e^{2} \mathbf{E}(s)}{m} d s-\int_{-\infty}^{t} d t^{\prime} \int_{-\infty}^{t^{\prime}} d s \chi\left(t^{\prime}-s\right) \mathbf{J}_{0}(s) .
$$

Hence the Laplace transform $\mathcal{L}(\sigma, \Omega)$ of the conductivity reads

$$
\mathcal{L}(\sigma, \Omega)=\mathcal{L}\left(\sigma_{0}, \Omega\right)+\mathcal{L}\left(\sigma_{1}, \Omega\right),
$$

where the correction to the model conductivity $\sigma_{1}$ is given by

$$
\mathcal{L}\left(\sigma_{1}, \Omega\right)=\frac{e^{2}}{m} \frac{v^{2}-w^{2}}{\Omega\left(v^{2}+\Omega^{2}\right)}-\frac{\mathcal{L}\left(\sigma_{0}, \Omega\right) \mathcal{L}(\chi, \Omega)}{\Omega} .
$$

A more accurate conductivity can be found using the standard resummation argument,

$$
\mathcal{L}(\sigma, \Omega)=\mathcal{L}\left(\sigma_{0}, \Omega\right)\left(1+\frac{\mathcal{L}\left(\sigma_{1}, \Omega\right)}{\mathcal{L}\left(\sigma_{0}, \Omega\right)}\right) \approx \frac{\mathcal{L}\left(\sigma_{0}, \Omega\right)}{1-\frac{\mathcal{L}\left(\sigma_{1}, \Omega\right)}{\mathcal{L}\left(\sigma_{0}, \Omega\right)}},
$$

that is, expression (38) in FHIP. Consequently the dc conductivity for the optical Fröhlich polaron reads

$$
\sigma_{d c}=\lim _{\Omega \rightarrow 0} \mathcal{L}(\sigma, \Omega)=\frac{w^{2}}{v^{2}} \frac{3 e^{2}}{2 \alpha m \omega_{L O} n_{B}\left(\omega_{L O}\right)} .
$$

Moreover, since $v^{2} / w^{2}=m^{*} / m$ [6] we have

$$
\sigma_{d c}=\frac{3 e^{2}}{2 \alpha m^{*} \omega_{L O} n_{B}\left(\omega_{L O}\right)} \approx \frac{3 e^{2}}{2 \alpha m^{*} \omega_{L O}} e^{\beta \hbar \omega_{L O}},
$$

consistent with our result (4.4). 
[1] H. Fröhlich, Proc. R. Soc. London, Ser. A 160, 230 (1937).

[2] H. Fröhlich, H. Pelzer, and S. Zienau, Philos. Mag. 41, 221 (1950).

[3] H. Fröhlich, Adv. Phys. 3, 325 (1954).

[4] A. S. Alexandrov and J. T. Devreese, Advances in Polaron Physics (Springer-Verlag, Berlin, 2010).

[5] J. T. Devreese, arXiv:1012.4576v4.

[6] R. Feynman, R. Hellwarth, C. Iddings, and P. Platzman, Phys. Rev. 127, 1004 (1962).

[7] L. P. Kadanoff, Phys. Rev. 130, 1364 (1963).

[8] D. J. Howarth and E. H. Sondheimer, Proc. R. Soc. London, Ser A 219, 53 (1953).

[9] F. E. Low and D. Pines, Phys. Rev. 98, 414 (1955).

[10] Y. Osaka, Prog. Theor. Phys. 25, 517 (1961).

[11] F. M. Peeters and J. T. Devreese, Phys. Status Solidi B 115, 539 (1983).

[12] F. M. Peeters and J. T. Devreese, in Solid State Physics, edited by F. Seitz and D. Turnbull, Vol. 38 (Academic, New York, 1984), p. 81 .
[13] E. P. Wigner, Phys. Rev. 40, 749 (1932).

[14] R. P. Feynman and F. L. Vernon, Jr., Ann. Phys. (N.Y.) 24, 118 (1963).

[15] R. P. Feynman, Phys. Rev. 97, 660 (1955).

[16] D. Sels, F. Brosens, and W. Magnus, Physica A 392, 326 (2013).

[17] D. Sels and F. Brosens, Phys. Rev. E 88, 042101 (2013).

[18] J. T. Devreese and R. Evrard, Phys. Status Solidi B 78, 85 (1976).

[19] A. Polkovnikov, Ann. Phys. (N.Y.) 325, 1790 (2010).

[20] J. T. Devreese, J. De Sitter, and M. Goovaerts, Phys. Rev. B 5, 2367 (1972).

[21] G. N. A. Watson, Treatise on the Theory of Bessel Functions, 2nd ed. (Cambridge University Press, Cambridge, 1966), Chap. XIII.

[22] V. F. Los', Theor. Math. Phys. 60, 703 (1984).

[23] G. De Filippis, V. Cataudella, A. S. Mishchenko, C. A. Perroni, and J. T. Devreese, Phys. Rev. Lett. 96, 136405 (2006).

[24] S. Klimin and J. T. Devreese, arXiv:1310.4413 [Phys. Rev. B (to be published)]. 